\title{
Infección por SARS-CoV-2 y obesidad
}

\section{SARS-CoV-2 infection and obesity}

\author{
Rosero $R J^{1}$, Ramírez $A^{2}$, Pinzón $J B^{3}$
}

${ }^{1}$ Internista y Endocrinólogo. Clínica de Obesidad, Dismetabolismo y Deporte (COD2). Clínica Las Américas, Medellín, Colombia. ORCID ID: https://orcid.org/0000-0003-4384-8629.

${ }^{2}$ Internista y Endocrinólogo. Universidad Pontificia Bolivariana, Clínica Intergral de Diabetes, Medellín, Colombia.

${ }^{3}$ Internista, Endocrinólogo y Epidemiólogo Clínico. Centro Médico Carlos Ardila Lulle, Bucaramanga, Colombia.

Autor de correspondencia: Ricardo J. Rosero Revelo

Correo electrónico: cod2@clincalasamericas.com.co

\section{Resumen}

La pandemia por coronavirus 2019 (COVID-19) ha tenido diferentes efectos. Uno de ellos fue concentrar la atención de múltiples investigadores y clínicos para dedicarse a estudiar y tratar de entender los retos de esta infección en diferentes poblaciones, que se han determinado en riesgo de presentar una manifestación más grave de la infección. La obesidad ha sido recientemente identificada como una de las comorbilidades de mayor riesgo junto con la insuficiencia cardíaca, con una probabilidad siete veces mayor para el requerimiento de ventilación mecánica invasiva. La obesidad es un factor en la gravedad de la enfermedad del SARS-CoV-2, que tiene un mayor impacto en pacientes con un índice de masa corporal (IMC) $\geq 35 \mathrm{~kg} / \mathrm{m}^{2}$. Los pacientes con obesidad, especialmente aquellos con grado severo, deben tomar medidas adicionales para evitar el contagio con SARS-CoV-2, mediante el cumplimiento de las medidas de prevención durante la pandemia actual, si se quiere, de forma más rigurosa.

Palabras clave: obesidad, COVID-19.

\section{Abstract}

The 2019 coronavirus pandemic (COVID-19) has had different effects. One of them was to focus the attention of multiple researchers and clinicians to dedicate themselves to studying and trying to understand the challenges of this infection in different populations, which have been determined in risk of presenting a more severe manifestation of the infection. Obesity has recently been identified as one of the highest-risk comorbidities along with heart failure, with a 7-fold greater probability of requiring mechanical ventilation.
Obesity is a factor in the severity of SARS-CoV-2 disease, which has a greater impact in patients with a body mass index (BMI) $\geq 35 \mathrm{~kg} / \mathrm{m}^{2}$. Obese patients, especially those with a severe degree, must take additional measures to avoid contagion with SARS-CoV-2, and must comply with preventive measures during the current pandemic, if desired, more rigorously.

Keywords: Obesity, COVID-19.

\section{Introducción}

La pandemia por el coronavirus SARS-CoV-2 (COVID-2019) ha tenido diferentes efectos. Uno de ellos fue desviar, momentáneamente, el foco de múltiples áreas de investigación para dedicarse a estudiar y tratar de entender los retos de esta infección. De esta manera, se reconocieron diferentes poblaciones en riesgo de presentar una manifestación de la infección más grave. En un inicio, se identificaron ciertas morbilidades que se asociaron a un mayor riesgo de desenlaces negativos; entre ellas, hipertensión y otras enfermedades cardiovasculares, enfermedades respiratorias crónicas, diabetes, enfermedad renal, enfermedad hepática, inmunocompromiso y, además, grupo etario de mayores de 65 años ${ }^{(1)}$.

Los reportes iniciales de China (en particular de Wuhan) e Italia (en especial de la región de Lombardía) informaron las condiciones comórbidas asociadas a la COVID-19 relacionadas con la tasa más alta de mortalidad. Estas fueron la enfermedad cardiovascular (ECV) $(10,5 \%)$ y la diabetes mellitus $(7,3 \%)$, seguidas de las enfermedades respiratorias crónicas $(6,3 \%)$, la hipertensión $(6,0 \%)$ y el cáncer $\left(5,6 \%{ }^{(2)}\right.$; sin embargo, no proporcionaron datos sobre el peso corporal y la altura para su caracterización por índice de masa corporal (IMC).

Al mismo tiempo, en forma descriptiva, Puig-Domingo menciona la experiencia en algunos hospitales en España, en donde la coexistencia de obesidad grave en pacientes jóvenes favorecía la evolución hacia la destrucción alveolar, con insuficiencia respiratoria y muerte ${ }^{(3)}$. Cuando la pandemia llega a Estados Unidos, un estudio descriptivo, con una pequeña muestra de 24 pacientes críticos (63\% varones) diagnosticados con COVID-19 en Seattle, fue uno de los primeros en reportar datos de IMC ( 3 pacientes con un IMC en la categoría normal, 7 con sobrepeso, 13 con obesidad y 1 con datos fal- 
tantes), y se documentó que el $85 \%$ de los pacientes con obesidad requirieron ventilación mecánica y el 62\% fallecieron. Estas proporciones fueron mayores que las observadas en pacientes sin obesidad, de los cuales, el $64 \%$ requirió ventilación mecánica y el 36\% murió. Lo anterior generó una alerta para la búsqueda de la asociación de obesidad con un peor desenlace en pacientes con esta patología y determinar así si había correlación ${ }^{(4)}$.

\section{Relación entre obesidad y COVID-19}

La obesidad debe comprenderse como una patología crónica con determinantes genéticos y ambientales, que implica la acumulación de grasa, particularmente, a nivel visceral y en otros órganos (ectópica), y genera una activación interrelacionada de múltiples adipocinas y una respuesta de insulinorresistencia compensadora. Estos tres fenómenos suscitan, a su vez, un estado de inflamación latente y persistente en el individuo obeso, una alteración de la fibrinólisis, la elevación de las cifras de presión arterial y de la glucemia, entre otros procesos ${ }^{(5,6)}$. De igual forma, el tejido adiposo en obesidad es proinflamatorio, con una mayor expresión de citocinas, particularmente las adipocinas. También cursa con una expresión de leucocitos y macrófagos, que reemplaza el tejido regulador (M2). La obesidad per se es un factor de riesgo independiente y causal para el desarrollo de una enfermedad mediada por el sistema inmunitario ${ }^{(7)}$, lo que sugiere que dicho estado de adipositis puede tener consecuencias inmunitarias sistémicas tras una provocación ambiental adicional. En términos de defensa del huésped, la obesidad afecta las respuestas inmunitarias adaptativas al virus de la influenza ${ }^{(8)} \mathrm{y}$, posiblemente, podría hacerlo con el SARS-CoV-2. Las personas obesas pueden exhibir una mayor eliminación viral, lo que sugiere, a su vez, un potencial de gran exposición viral y, por ende, mayor carga. Esto podría facilitarse en hogares multigeneracionales superpoblados o en viviendas multifamiliares, que son más comunes en las comunidades socioeconómicamente desfavorecidas, en donde la obesidad es prevalente.

La obesidad se considera una condición de inflamación crónica leve, como ya se mencionó, y las interleucinas (IL) pueden influir en la función de las células grasas de diversas maneras. Las interleucinas proinflamatorias que aumentan en personas obesas, como el factor de necrosis tumoral alfa (FNT- $\alpha$ ), IL-1 $\alpha$ e IL- 6 secretadas por los macrófagos tipo I (MCP-1), inducen efectos indeseables que conducen a resistencia a la insulina y complicaciones cardiovasculares en pacientes con exceso de peso. El estrés del retículo endoplásmico asociado al aumento de la generación de radicales de oxígeno y la lipotoxicidad activan a los inhibidores kappa-beta (Ік $\beta$ ), que conducen, a su vez, a la activación del factor nuclear kappa $\mathrm{B}(\mathrm{FN}-\kappa \mathrm{B})$ en el tejido adiposo; de forma alterna, el aumento de la presencia de mediadores inflamatorios en el tejido adiposo puede aumentar la producción de las citocinas ${ }^{(9)}$.
En la infección por SARS-CoV-2, se ha observado una respuesta exagerada caracterizada, principalmente, por la secreción de citocinas proinflamatorias aberrantes asociada a gran cantidad de macrófagos alveolares, con disminución de células T-CD4+ y T-CD8+, pero no con células B, con sobreproducción de IL-6, IL-2R, IL-10 y FNT- $\alpha$, ni disminución de la expresión de interferón gamma (IFN- $\gamma$ ), lo que se ha correlacionado con la gravedad de la enfermedad. La manifestación más grave se conoce como tormenta de citocinas, que representa un episodio inflamatorio hiperagudo asociado a un pobre pronóstico en pacientes con COVID-19 y comparte algunas características con los hallazgos fisiopatológicos arriba descritos en el paciente con sobrepeso u obesidad ${ }^{(10)}$.

\section{Obesidad como condición de riesgo en COVID-19}

La obesidad o el exceso de masa de grasa ectópica puede ser un factor detonante de la infección grave por COVID-19, que reduce la reserva cardiorrespiratoria y potencia las alteraciones inmunológicas que podrían mediar la progresión a la enfermedad crítica y a falla orgánica en una proporción de pacientes con esta patología.

A nivel cardiometabólico, la obesidad está causalmente relacionada con hipertensión, diabetes, enfermedad coronaria, accidente cerebrovascular, fibrilación auricular, enfermedad renal e insuficiencia cardíaca ${ }^{(11,12)}$. Sin embargo, la obesidad ha sido descrita como un factor de predisposición independiente para la infección pulmonar H1N1 grave ${ }^{(13)}$, por ejemplo. Además, la obesidad abdominal se asocia con una ventilación deteriorada de la base de los pulmones, lo que resulta en una saturación reducida de oxígeno en la sangre. Así mismo, la inflamación crónica de bajo grado, característica de la obesidad abdominal, puede afectar la respuesta inmunitaria ${ }^{(6)} \mathrm{y}$ tener efectos sobre el parénquima pulmonar y los bronquios. Todo lo anterior puede explicar la asociación de obesidad con la gravedad de la enfermedad del SARS-CoV-2, la cual parece tener un mayor impacto en pacientes con un IMC $\geq 35 \mathrm{~kg} / \mathrm{m}^{2}$. Los pacientes con obesidad, especialmente aquellos con grado severo, deben ser muy estrictos en el cumplimiento general de las medidas de salud pública, confinamiento, distanciamiento físico, higiene exhaustiva, entre otros, para disminuir la probabilidad de contagio y el desarrollo de enfermedad por el nuevo coronavirus 2019.

\section{Evidencia de la relación entre obesidad y gravedad de la COVID-19}

A medida que nuevos reportes son publicados, se relaciona más estrechamente a la obesidad con la infección más grave por COVID-19 y con mayor riesgo de muerte.

En una evaluación retrospectiva que buscó evaluar la relación entre peso y requerimiento de admisión a un hospital en 
Nueva York, actualmente epicentro de la pandemia por COVID-19 en Estados Unidos, la Dra. Jennifer Lighter realizó un análisis estratificado por edad e IMC. En menores de 60 años, la presencia de obesidad grado 1 se asoció a un odds ratio (OR) de 2 (IC: 1,6-2,6) para requerir admisión hospitalaria y de 1,8 $(1,2-2,7)$ para requerir cuidados intensivos, con respecto a individuos de peso normal. Los pacientes con obesidad grado 2 (IMC >35) tenían un OR de 2,2 (1,7-2,9) y de 3,6 (2,5-5,3) para los mismos desenlaces, en comparación con personas de peso normal ${ }^{(14)}$. Aunque los pacientes menores de 60 años, generalmente, se consideran un grupo de menor riesgo de gravedad durante la COVID-19, la obesidad parece ser un factor de riesgo no reconocido previamente para el ingreso hospitalario y la necesidad de atención crítica en este grupo etario, lo que indica que la obesidad en personas jóvenes es un factor de riesgo epidemiológico que puede contribuir al aumento de las tasas de morbilidad experimentadas en este subgrupo de sujetos ${ }^{(14) .}$

En la misma ciudad, otra investigación evaluó a 7719 sujetos para infección por SARS-CoV-2, de los cuales, 4103 (48,7\%) fueron positivos para enfermedad por COVID-19. Las características poblacionales mostraron una mediana de edad de 52 años, $50,5 \%$ de género masculino. En cuanto a las comorbilidades, un total de 614 sujetos (15,0\%) tenían diabetes, 1100 (26,8\%) obesidad y 1235 (30,1\%) enfermedad cardiovascular. De igual forma, se evidenció en pacientes hospitalizados una duración media de estancia hospitalaria de 4,8 días (IC: 3,3-7,6) ${ }^{(15)}$.

Un estudio reciente, que incluyó 383 pacientes con COVID-19 atendidos en el Hospital de Shenzhen, en China, buscó determinar la asociación de obesidad con cuadros graves de enfermedad por SARS-CoV-2. Se encontró que el sobrepeso se asoció con un $86 \%$ más de riesgo y la obesidad con un 142\% más de riesgo para desarrollar neumonía grave en comparación con los pacientes de peso normal en modelos estadísticos que controlaron posibles factores de confusión ${ }^{(16)}$. En esa misma dirección, una cohorte retrospectiva de pacientes en Lille
(Francia) encontró que el 47\% de los pacientes admitidos eran obesos y la proporción de pacientes que requerían ventilación mecánica invasiva aumentaba por categorías de mayor IMC. El OR para requerir dicho soporte invasivo en pacientes con IMC mayor de 35 , con respecto a los de menos de 25 , era de 7,36 (1,63-33), luego de ajustar para otras variables de riesgo ${ }^{(17)}$.

\section{Conclusiones}

Los anteriores hallazgos, ya evidenciados en otras pandemias, permiten aconsejar medidas de prevención para la población con obesidad, aún jóvenes y sin otros factores de riesgo, dado un curso potencialmente más ominoso de la enfermedad en ellos. Así mismo, en aquellos pacientes obesos que la desarrollen, una observación activa más estrecha y minuciosa debe ser instaurada, dada su facilidad para desarrollar complicaciones más graves e incluso morir.

Corroborando lo mencionado por el Dr. Stefan ${ }^{(18)}$, la obesidad es un factor de riesgo principal para esta enfermedad, al igual que para un deterioro de la salud metabólica (como dislipidemia y resistencia a la insulina) y también está relacionada con un mayor riesgo de neumonía. La medición de las características antropométricas y los parámetros metabólicos son cruciales para estimar mejor el riesgo de complicaciones en pacientes con COVID-19.

\section{Conflicto de interés}

Ninguno.

\section{Financiación}

Ninguna.

\section{Agradecimientos}

Queremos agradecer a la Asociación Colombiana de Endocrinología por el compromiso y apoyo continuo en la educación y participación científica en temas de alta relevancia.

\section{Referencias}

1. Centers for Disease Control and Prevention. Coronavirus Disease 2019 (COVID-19). Group at Higher Risk for Severe Illness [Internet]. 2020. Disponible en: https://bit.ly/2M9wLcG.

2. Wu Z, McGoogan JM. Characteristics of and important lessons from the coronavirus disease 2019 (COVID-19) outbreak in China: summary of a report of 72,314 cases from the Chinese Center for Disease Control and Prevention. JAMA. 2020;323(13):1239-42. doi:10.1001/jama.2020.2648.

3. Puig-Domingo M, Marazuela M, Giustina A. COVID-19 and endocrine diseases. A statement from the European Society of Endocrinology. Endocrine. 2020;68(1):2-5. doi: 10.1007/s12020-020-02294-5.

4. Bhatraju PK, Ghassemieh BJ, Nichols M, Kim R, Jerome KR, Nalla AK, et al. Covid-19 in critically ill patients in the Seattle Region - Case Series. N Engl J Med. 2020;382(21):2012-2022. doi: 10.1056/ NEJMoa2004500.

5. Atawia RT, Bunch KL, Toque HA, Caldwell RB, Caldwell RW. Mechanisms of obesity-induced metabolic and vascular dysfunctions. Front Biosci (Landmark Ed). 2019;24:890-934.
6. Samad F, Ruf W. Inflammation, obesity, and thrombosis, Blood. 2013;122(20):3415-22. doi: 10.1182/blood-2013-05-427708.

7. Budu-Aggrey A, Brumpton B, Tyrrell J, Watkins S, Modalsli EH, CelisMorales C, et al. Evidence of a causal relationship between body mass index and psoriasis: A Mendelian randomization study. PLoS Med. 2019;16(1):e1002739. doi: 10.1371/journal.pmed.1002739.

8. Green WD, Beck MA. Obesity Impairs the Adaptive Immune Response to Influenza Virus. Ann Am Thorac Soc. 2017;14(Suppl 5):S406-S409. doi: 10.1513/AnnalsATS.201706-447AW.

9. Rosero RJ, Polanco JP, Sánchez P, Hernández E, Pinzón JB, Lizcano F. Obesidad: un problema en la atención de Covid-19. Rev Rep Med Cir. 2020. doi: https://doi.org/10.31260/RepertMedCir.01217372.1035.

10. Wang W, He J, Lie P, Huang L, Wu S, Lin Y, et al. The definition and risks of cytokine release syndrome-like in 11 COVID-19-infected pneumonia critically ill patients: disease characteristics and retrospective analysis. medRxiv. 2020. doi: https://doi.org/10.1101/2020.02.26.20026989. 
11. Csige I, Ujvárosy D, Szabó Z, Lőrincz I, Paragh G, Harangi M, et al. The Impact of Obesity on the Cardiovascular System. J Diabetes Res. 2018;2018:3407306. doi: 10.1155/2018/3407306.

12. Sattar N, McInnes IB, McMurray JJV. Obesity a Risk Factor for Severe COVID-19 Infection: Multiple Potential Mechanisms. Circulation. 2020. doi: 10.1161/CIRCULATIONAHA.120.047659.

13. Louie JK, Acosta M, Winter K, Jean C, Gavali S, Schechter R, et al. Factors associated with death or hospitalization due to pandemic 2009 influenza A(H1N1) infection in California. JAMA 2009;302(17):1896-902. doi: 10.1001/jama.2009.1583.

14. Lighter J, Phillips M, Hochman S, Sterling S, Johnson D, Francois F, et al. Obesity in patients younger than 60 years is a risk factor for Covid-19 hospital admission. Clin Infect Dis. 2020; ciaa415. doi: 10.1093/cid/ciaa415.
15. Petrilli CM, Jones SA, Yang J, Rajagopalan $\mathrm{H}$, O'Donnell LF, Chernyak Y, et al. Factors associated with hospitalization and critical illness among 4,103 patients with Covid-19 disease in New York City. mdeRxiv. 2020. doi: https://doi.org/10.1101/2020.04.08.20057794.

16. Qingxian C, Chen F, Wang T, Luo F, Liu X, Wu Q, et al. Obesity and COVID-19 severity in a designated hospital in Shenzhen, China. Diabetes Care 2020;dc200576. doi: 10.2337/dc20-0576.

17. Simonnet A, Chetboun M, Poissy J, Raverdy V, Noulette J, Duhamel A, et al. High prevalence of obesity in severe acute respiratory syndrome coronavirus-2 (SARS-CoV-2) requiring invasive mechanical ventilation. Obesity. 2020. doi: 10.1002 /oby.22831.

18. Stefan N, Birkenfeld AL, Schulze MB, Ludwig DS. Obesity and impaired metabolic health in patients with COVID-19. Nat Rev Endocrinol. 2020;1 2. doi: 10.1038/s41574-020-0364-6. 